\title{
图解中心原子轨道的杂化方式
}

赵苹苹 $1,{ }^{*}$, 蔡苹 2 , 胡锴 2 , 罗威 ${ }^{2}$, 程功臻 $2,{ }^{*}$

1 武汉大学印刷与包装系, 武汉 430072

2 武汉大学化学与分子科学学院, 武汉 430072

摘要: 借助图形方法, 分析了不同常见几何构型分子中心原子轨道杂化方式的原因一一端基原子轨道的有效重叠, 利用中心原子价层轨道的能量接近度的判断, 决定最终可能的杂化方式。

关键词: 杂化; 原子轨道; 分子结构; 图解法

中图分类号: G64; O6

\section{A Graphical Explanation of Atomic Orbital Hybridization}

\author{
Pingping Zhao ${ }^{1, *}$, Ping Cai ${ }^{2}$, Kai Hu ${ }^{2}$, Wei Luo ${ }^{2}$, Gongzhen Cheng ${ }^{2,{ }^{*}}$ \\ ${ }^{1}$ School of Printing and Packaging, Wuhan University, Wuhan 430072, P. R. China. \\ ${ }^{2}$ College of Chemistry and Molecular Sciences, Wuhan University, Wuhan 430072, P. R. China.
}

\begin{abstract}
For molecules with common geometries, a graphical method of effective overlap between terminal atomic orbitals and the valance-shell orbitals of the central atom was described; the energy adjacencies of these orbitals of the central atom were used to determine which orbitals would participate in orbital hybridization.
\end{abstract}

Key Words: Hybridization; Atomic orbitals; Molecular structure; Graphical method

杂化轨道理论是化学键的价键理论不可或缺的骨干, 是现代化学键理论的重要组成部分。相对 于分子轨道理论而言, 价键理论更符合我们对于化学键的直觉, 理解难度小, 因而非常容易被人接 受。然而, 大多数基础化学教科书里 ${ }^{[1]}$, 对各类不同空间结构分子的中心原子所采用特定杂化方式 背后的原因, 并无简单易懂的解释, 大多数的教学过程中与价层电子对互斥理论相结合, 直接交代 不同电子对构型分子或离子对应的中心原子杂化方式: 直线形电子对排列的是 $s p$ 杂化, 三角形的 $s p^{2}$ 杂化, 四面体的 $s p^{3}$ 杂化等 ${ }^{[2-5]}$ 。学生主要靠记忆实现这部分知识的获取。

对中心原子杂化方式的解释, 一般基于群表示理论 ${ }^{[6,7]}$, 但对于基础化学阶段的学生而言, 理解 起来相对困难。本文试图将定性多原子分子轨道中常使用的配体群轨道与中心原子轨道相适配的理 念用于对中心原子杂化方式的理解, 从笔者多年的教学实践效果来看, 非常容易获得学生的接受, 值得一试。

\section{1 图形方法的基本思想}

价键理论中化学键的形成基于价层原子轨道的重叠, 杂化轨道理论是对价键理论的拓展, 中心 原子的价层轨道为了适应不同空间结构的需求预先混合, 形成适应结构需求的中心原子杂化原子轨

收稿: 2020-07-20; 录用: 2020-07-22; 网络发表: 2020-07-27 
道, 该理论认为结构决定杂化方式, 杂化发生在中心原子的价层轨道内, 杂化后的中心原子杂化轨 道与端基原子价层轨道重叠, 从而形成各类化学键。分子轨道理论则用形成分子的所有原子的所有 原子轨道的线性组合构建分子轨道, 为了简化计算, 可以按照中心原子轨道对称性需求将端基原子 轨道进行线性组合, 形成配体群轨道, 该过程同样依赖对称群表示的特征标表, 与杂化轨道理论的 需求有相似性。可以将该思想用于中心原子价层轨道的杂化。

\section{2 成键与非键重叠}

一般教科书介绍价键理论的时候, 只介绍 $s 、 p$ 轨道之间的重叠, 为了讨论的需要, 必须将 $d$ 轨 道的重叠加以考虑。除了考虑 $\sigma$ 成键重叠、 $\pi$ 成键重叠, 还必须考虑在价键理论一般不作介绍的非 键重叠。注意决定分子几何结构的是 $\sigma$ 键, 因而, 探究中心原子采用何种杂化方式的内在原因的时 候只需要关注 $\sigma$ 重叠方式和非键重叠。

\section{1 包含 $\boldsymbol{d}$ 轨道的 $\boldsymbol{\sigma}$ 重叠}

关于 $s 、 p 、 d$ 轨道之间的成键 $\sigma$ 重叠，如图 1 所示。

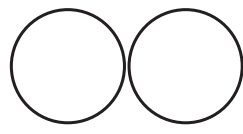

$S-S$ 成键 $\sigma$ 重叠

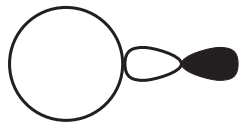

$S-p$ 成键 $\sigma$ 重叠

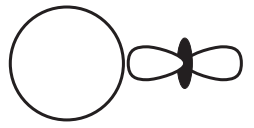

$S-d$ 成键 $\sigma$ 重叠

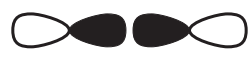

$p-p$ 成键 $\sigma$ 重叠

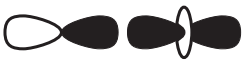

$p-d$ 成键 $\sigma$ 重叠

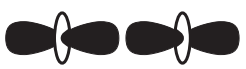

$d-d$ 成键 $\sigma$ 重叠

图 $1 s 、 p 、 d$ 轨道之间的成键 $\sigma$ 重叠

\section{2 包含 $\boldsymbol{d}$ 轨道的 $\boldsymbol{\pi}$ 重叠}

关于 $p 、 d$ 轨道之间的成键 $\pi$ 重叠，如图 2 所示。

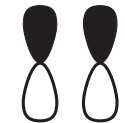

$p-p$ 成键 $\pi$ 重叠

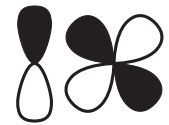

$p$ - $d$ 成键 $\pi$ 重叠

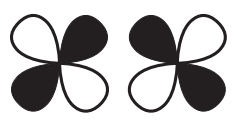

$d-d$ 成键 $\pi$ 重叠

图 $2 p 、 d$ 轨道之间的成键 $\pi$ 重叠

\section{3 包含 $\boldsymbol{d}$ 轨道的非键重叠}

非键重叠不会形成化学键, 如图 3 所示。 


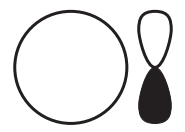

$s-p$ 非键重叠

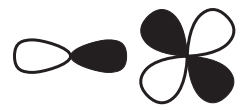

$p-d$ 非键重叠

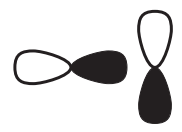

$p-p$ 非键重叠

图 $3 s, p 、 d$ 轨道之间的非键重叠

\section{3 中心原子的价层轨道杂化}

杂化轨道理论在讨论原子轨道杂化时实际上是忽略了不同原子轨道的能量差异的。把观察问题 的中心放在了对称性匹配与否上。只有对称性与形成分子空间结构相一致的原子轨道才有机会参与 杂化。

\section{1 直线形分子中心原子的杂化}

为了讨论方便起见, 如图 4 所示, 将分子 $\mathrm{ET}_{2}$ 置于 $z$ 坐标轴上, 中心原子 $\mathrm{E}$ 置于原点, 两个端基原子 $\mathrm{T}$ 分别位于 $z$ 轴的正负方向, 与 原点距离相同。

将端基原子的价层轨道简化为图 5 所示的样式, 其相位随中心原 子的需求而任意改变, 可以为正, 亦可为负, 而且永远指向中心原子。

假定中心原子的价层轨道包含 $s 、 p$ 和 $d$ 轨道, 它们的简并轨道 数分别为 $1 、 3$ 和 5 。总原子轨道数为 9 。通过穷举法以及简单的图像 分析, 能够发现中心原子的 $s$ 轨道、 $p_{z}$ 轨道和 $d_{z^{2}}$ 轨道均可以端基原 子轨道之间发生有效的 $\sigma$ 成键重叠, 如图 6 所示。

而中心原子价层轨道中其他剩余的 6 个轨道与端基原子轨道之 间均为非键重叠!

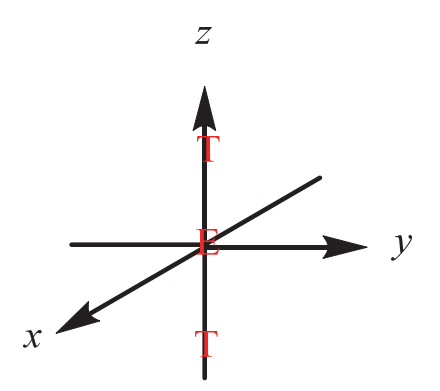

图 4 直线形分子的空间示意图

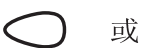

图 5 端基原子价层轨道简化图
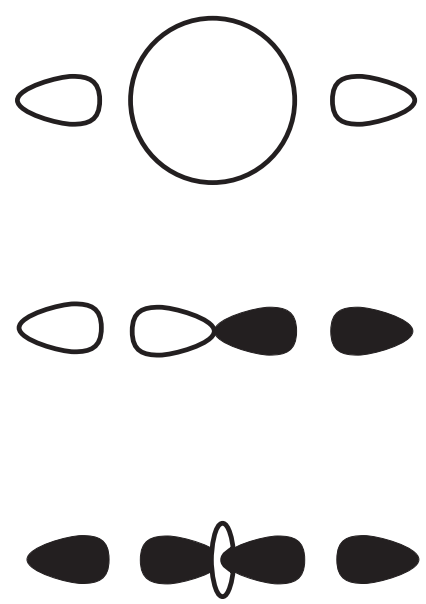

图 6 直线型分子内中心原子与端基原子价层轨道之间的成键 $\sigma$ 重叠 
因而, 如果不考虑轨道之间的能级差异, 中心原子的 $s$ 轨道、 $p_{z}$ 轨道和 $d_{z^{2}}$ 轨道均有可能参与杂 化。

形成杂化轨道的总数总是等于参与杂化的原子轨道数的总和。

形成直线形分子, 需要形成两个 $\sigma$ 键, 也就是需要两个杂化轨道。

我们需要从以上中心原子的 $s$ 轨道、 $p_{z}$ 轨道和 $d_{z^{2}}$ 轨道中选择其中任意两个的组合就可能形成两 个杂化轨道。也就是 $s p$ 杂化、 $d s$ 杂化或 $p d$ 杂化都有可能。

具体参与杂化的原子轨道是哪些取决于中心原子价层轨道的能级差, 能量最接近的两个轨道杂 化应该是对称性和能量接近都能满足的杂化方式。

对于中心原子是第二、三周期元素的直线形化合物来说，只可能是 $s p$ 杂化方式。

对于中心原子是第四或更大周期元素的直线形化合物来说，有可能是 $d s$ 杂化!

受镧系收缩影响的中心原子的直线形化合物来说, 有可能发生 $p d$ 杂化。

\section{2 平面三角形分子中心原子的杂化}

将分子 $\mathrm{ET}_{3}$ 置于 $x z$ 平面内, 中心原子 $\mathrm{E}$ 置于原点, 其中一个 端基原子 $\mathrm{T}$ 位于 $z$ 轴的正方向, 另外两个端基原子 $\mathrm{T}$ 分别位于该平 面的第三象限和第四象限, 并与 $x$ 轴成 30 度夹角, 如图 7 所示。

在平面三角形分子中，端基原子轨道与中心原子 $s 、 p$ 和 $d$ 轨 道中能发生有效(没有完全抵消) $\sigma$ 重叠的有图 8 所示的几种方式。

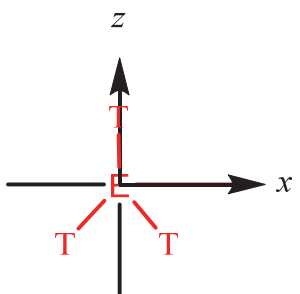

图 7 平面三角形分子的空间示意图
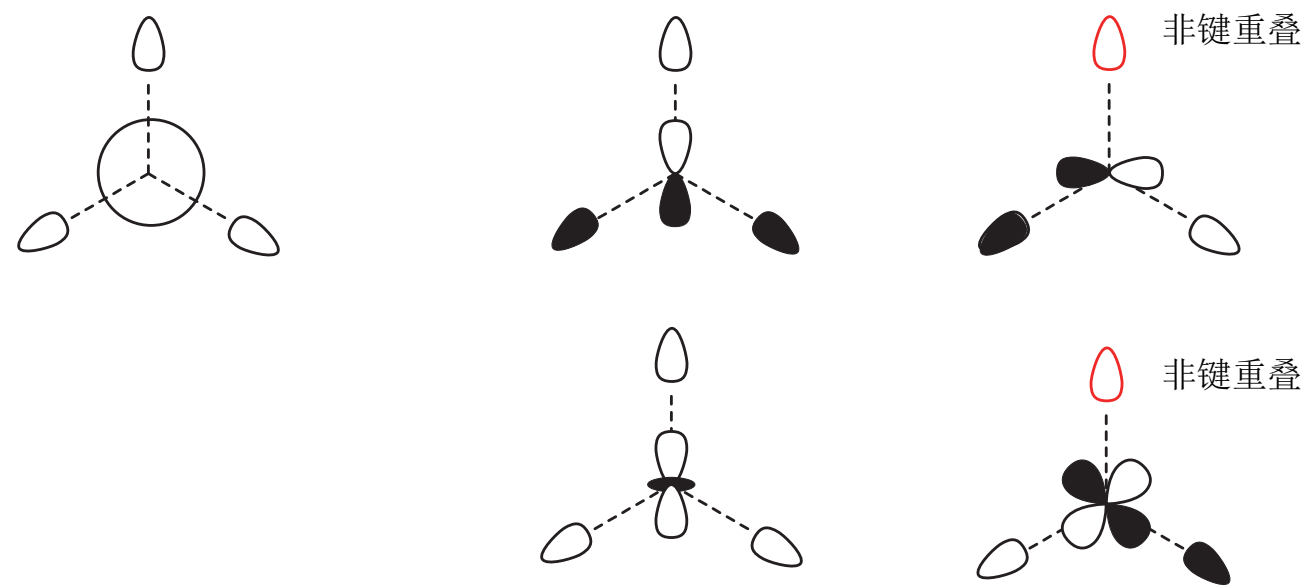

图 8 平面三角型分子内中心原子与端基原子价层轨道之间的有效重叠

即能够发生有效 $\sigma$ 重叠的中心原子轨道有 $s$ 轨道、 $p_{x}$ 轨道、 $p_{z}$ 轨道、 $d_{z^{2}}$ 轨道和 $d_{x z}$ 轨道。

同样的办法判断, 第二、三周期元素为中心原子时 $s p^{2}$ 杂化, 大于三周期元素作为中心原子时 $d^{2} s$ 杂化。

\section{3 四面体形分子中心原子的杂化}

将分子 $\mathrm{ET}_{4}$ 置于立方体内, 中心原子 $\mathrm{E}$ 置于原点, 四个端基原 子 $\mathrm{T}$ 分别占据立方体的四个顶点, 如图 9 所示。

从图 10 可以发现, 中心原子的 $s$ 轨道、三个 $p$ 轨道均可形成 有效的 $\sigma$ 重叠, 同样 $d_{x y} 、 d_{y z}$ 和 $d_{x z}$ 轨道亦可与端基原子轨道之间

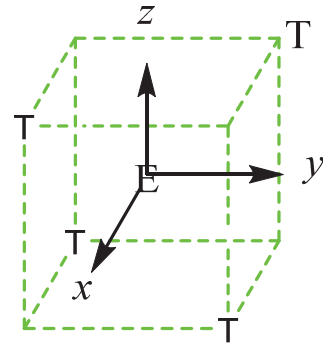

图 9 四面体分子的空间示意图 发生有效的 $\sigma$ 重叠。 

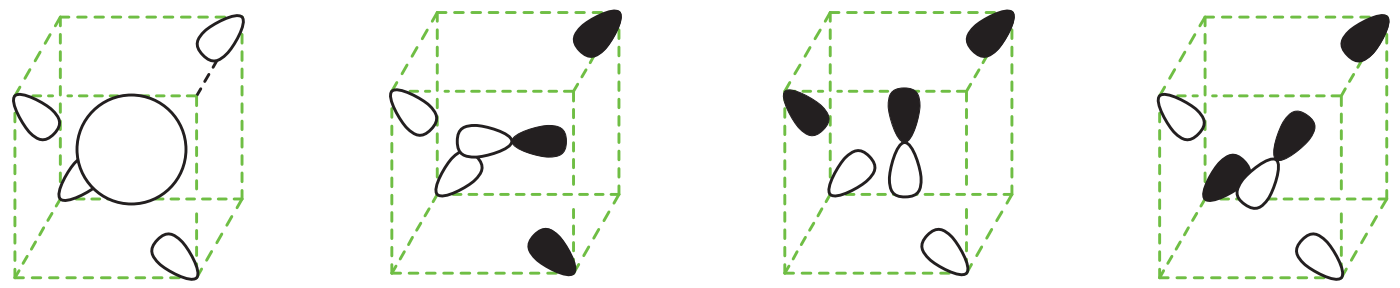

图 10 四面体型分子内中心原子与端基原子价层轨道之间的有效重叠

故而, 四面体的杂化方式可以有 $s p^{3} 、 d^{3} s$ 等。

\section{4 三角双雉形分子中心原子的杂化}

三角双雉可以看作是平面三角形与直线形的叠加, 注意叠加时中心原子的轨道不能重复使用, 因为其价层轨道总数是固定的, 杂化方式就成了 $s p^{2}+d p$, 也就是 $d s p^{3}$ 。不可能是 $s p^{2}+s p=s^{2} p^{3}$ !

3.5 平面正方形分子中心原子的杂化

将端基原子置于 $x, y$ 轴的正负方向上。

同样的办法推测可以形成有效 $\sigma$ 重叠的中心原子轨道有 $s$ 轨道、 $p_{x}$ 轨道、 $p_{y}$ 轨道, $d_{x^{2}-y^{2}}$ 轨道。 故而可能的杂化方式为 $d s p^{2}$ 杂化。

\section{6 八面体形分子中心原子的杂化}

可以将八面体看作是平面四方形与直线形的叠加, 所以杂化方式为 $d s p^{2}+d p=d^{2} s p^{3}$ 杂化。

\section{7 不等性杂化}

重叠程度偏离理想结构的均为不等性杂化, 其中重叠程度提高者杂化中参与成分提高, 而减弱 者则参与度减小。

\section{4 结语}

借助多原子分子的定性分子轨道处理的图形方法, 分析了不同几何构型分子中心原子轨道杂化 方式的原因一一与端基原子轨道的有效 $\sigma$ 重叠, 利用中心原子价层轨道的能量接近度的判断, 决定 最终可能的杂化方式。本方法相对简单明了, 不需要群论的基础。

\section{参 考 文 献}

\footnotetext{
[1] 宋天佑, 程鹏, 徐家宁, 张丽荣. 无机化学(上). 第 4 版. 北京: 高等教育出版社, 2019.

[2] 刘冰, 张丕俭. 大学化学, 2009, 24 (6), 29.

[3] 蔡苹, 胡锴, 程功榛. 大学化学, 2016, 31 (12), 69 .

[4] 吴集贵, 曾正志, 姚卡玲. 大学化学, 1991, 6(6), 9.

[5] 李小平. 大学化学, 1987, 2 (6), 39.

[6] Kimball, G. E. J. Chem. Phys. 1940, 8, 188.

[7] 弗格森, J. E. 无机立体化学和化学键. 刘举正, 俞国祯, 肖良质, 译. 北京: 高等教育出版社, 1983: 44-53.
} 